\title{
Childhood autism: translation and validation of the Childhood Autism Rating Scale for use in Brazil
}

\author{
Autismo infantil: tradução e validação da Childhood Autism Rating Scale \\ para uso no Brasil
}

\author{
Alessandra Pereira ${ }^{1}$, Rudimar S. Riesgo ${ }^{2}$, Mario B. Wagner ${ }^{3}$
}

\section{Resumo}

Objetivo: Traduzir, adaptar e validar uma versão em português (do Brasil) da Childhood Autism Rating Scale (CARS).

Métodos: Após processo de tradução, a versão foi aplicada em 60 pacientes com diagnóstico de autismo infantil, de 3 a 17 anos de idade, selecionados consecutivamente de um ambulatório especializado a fim de analisar as propriedades psicométricas da nova versão (CARS-BR) (consistência interna, validade e confiabilidade).

Resultados: A consistência interna foi elevada, com valor de alfa de Cronbach de 0,82; a validade convergente (comparada com a Escala de Avaliação de Traços Autístícos) alcançou um coeficiente de correlação de Pearson de $r=0,89$. Ao ser correlacionada à Escala de Avaliação Global de Funcionamento (para determinação da validade discriminante), a CARS-BR apresentou um coeficiente de correlação de Pearson de $r=-0,75$. A confiabilidade teste-reteste foi 0,90 .

Conclusão: A metodologia utilizada e os cuidados no processo de tradução permitem concluir que esse é um instrumento válido e confiável para avaliação da gravidade do autismo no Brasil.

J Pediatr (Rio J). 2008;84(6):487-494: Autismo infantil, estudos de validação, questionários.

\section{Introdução}

Embora Bleuler, em 1911, tenha sido o primeiro a descrever o autismo, foi Leo Kanner, em 1943, que o definiu a partir da observação de um grupo de crianças com comportamento peculiar caracterizado por uma incapacidade inata de estabelecer contato afetivo e interpessoal ${ }^{1-3}$.

O autismo é um transtorno invasivo do desenvolvimento, e seu quadro comportamental é composto basicamente de quatro manifestações: déficits qualitativos na interação social,

\section{Abstract}

Objective: To translate the Childhood Autism Rating Scale into Brazilian Portuguese and to determine the initial psychometric properties of the resulting version (CARS-BR).

Methods: The methodology used to produce an adequate version included translation, backtranslation and evaluation of semantic equivalence. In order to determine its psychometric properties (internal consistency, validity and reliability), the CARS-BR was administered to 60 consecutive patients with autism, aged between 3 and 17 years and seen at a university hospital.

Results: Internal consistency was high, with a Cronbach's alpha of 0.82. Convergent validity, in comparison with the Autistic Traits Assessment Scale, exhibited a Pearson's correlation coefficient of $r$ $=0.89$. When correlated with the Global Assessment of Functioning Scale in order to evaluate discriminant validity, the CARS-BR exhibited a Pearson's coefficient of $r=-0.75$. Test-retest reliability exhibited a kappa coefficient of 0.90 .

Conclusion: These results suggest that the CARS-BR is a valid and reliable instrument for evaluating autism severity in Brazil.

J Pediatr (Rio J). 2008;84(6):487-494: Autism, validation studies, questionnaires.

déficits na comunicação, padrões de comportamento repetitivos e estereotipados e um repertório restrito de interesses e atividades ${ }^{4}$. Somando-se aos sintomas principais, crianças autistas freqüentemente apresentam distúrbios comportamentais graves, como automutilação e agressividade em resposta às exigências do ambiente, além de sensibilidade anormal a estímulos sensoriais ${ }^{3,5}$. Apesar de décadas de pesquisas e investigações, a etiologia do autismo permanece indefinida, pois se trata de um distúrbio complexo e heterogêneo com graus variados de severidade ${ }^{3,5}$. Várias regiões

1. Mestre. Neurologista pediátrica, Universidade Federal do Rio Grande do Sul (UFRGS), Porto Alegre, RS.

2. Doutor.Neurologista pediátrico, Professor adjunto, Departamento de Pediatria, Faculdade de Medicina, UFRGS, Porto Alegre, RS.

3. PhD, University of London, London, UK. Professor adjunto, Departamento de Medicina Social, Programa de Pós-Graduação em Pediatria, Faculdade de Medicina, UFRGS, Porto Alegre, RS.

Apoio financeiro: Fundação Instituto de Pesquisas Econômicas.

Não foram declarados conflitos de interesse associados à publicação deste artigo.

Como citar este artigo: Pereira A, Riesgo RS, Wagner MB. Childhood autism: translation and validation of the Childhood Autism Rating Scale for use in Brazil. J Pediatr (Rio J). 2008;84(6):487-494.

Artigo submetido em 10.03.08, aceito em 06.08.08.

doi:10.2223/JPED. 1828 
cerebrais podem estar envolvidas no processo de desenvolvimento da patologia, incluindo cerebelo, hipocampo, amígdala, gânglios da base e corpo caloso, porém as anormalidades celulares e metabólicas, base para o desenvolvimento cerebral anormal, permanecem desconhecidas $^{6,7}$. O progresso na compreensão da causa, natureza e tratamento do autismo requer uma integração cada vez maior entre conceitos, achados genéticos, avanços na neurociência cognitiva e observações clínicas ${ }^{3,5,6}$.

A prevalência do autismo varia de 4 a 13/10.000, ocupando o terceiro lugar entre os distúrbios do desenvolvimento infantil à frente das malformações congênitas e da síndrome de Down ${ }^{3,8}$. Nos EUA, de cada 1.000 crianças nascidas, pelo menos uma irá, em algum momento do seu desenvolvimento, receber o diagnóstico de transtorno do espectro autista ${ }^{5,8}$. Na ausência de um marcador biológico, o diagnóstico de autismo permanece clínico. Os critérios atualmente utilizados são aqueles descritos no Manual Estatístico e Diagnóstico da Associação Americana de Psiquiatria, o Diagnostic and Statistical Manual of Mental Disorders (DSMIV) ${ }^{4}$.Os critérios do DSM-IV para diagnóstico de autismo têm um grau elevado de especificidade e sensibilidade em grupos de diversas faixas etárias e entre indivíduos com habilidades cognitivas e de linguagem distintas; no entanto, para avaliar os sintomas de forma quantitativa e refinar o diagnóstico diferencial, outros instrumentos são necessários ${ }^{9}$. A Childhood Autism Rating Scale (CARS) foi desenvolvida ao longo de 15 anos e é especialmente eficaz na distinção de casos de autismo leve, moderado e grave, além de discriminar crianças autistas daquelas com retardo mental ${ }^{10-14}$. Seu uso oferece diversas vantagens sobre outros instrumentos: a inclusão de itens que representam critérios diagnósticos variados e refletem a real dimensão da síndrome, aplicabilidade em crianças de todas as idades, inclusive pré-escolares, além de escores objetivos e quantificáveis baseados na observação direta ${ }^{10}$. A identificação do autismo é de fundamental importância, e a utilização de um instrumento padronizado e mundialmente aceito permite diagnóstico precoce e acurado, além de possibilitar a troca de informações entre diferentes centros de pesquisa ${ }^{14-16}$.

Neste estudo, objetivamos traduzir para a língua portuguesa, adaptar e validar a CARS.

\section{Métodos \\ População}

O estudo foi conduzido de setembro de 2006 a abril de 2007 no Ambulatório de Transtornos Invasivos do Desenvolvimento do Hospital de Clínicas de Porto Alegre. Participaram do estudo crianças e adolescentes de 3 a 17 anos, em acompanhamento no ambulatório, com diagnóstico de autismo primário pelo DSM-IV(critério de inclusão). O tamanho amostral foi calculado considerando o coeficiente de Cronbach com uma margem de erro máxima de 0,1. Utilizando $\alpha=0,05$, estimou-se que seriam necessários 60 pacientes com autismo para a fase de validação. A versão da CARS traduzida para o português (CARS-BR), a Escala de Avaliação de Traços Autísticos (ATA) e a Escala de Avaliação Global do Funcionamento (GAF) foram aplicadas em uma amostra consecutiva de 60 pacientes. A inclusão consecutiva ocorreu por ordem de chegada para consulta de rotina no ambulatório. A CARS-BR foi reaplicada em 50 pacientes desta amostra definidos por sorteio.

Todos os responsáveis legais foram informados sobre os objetivos da pesquisa e um consentimento informado para cada participante foi obtido. Este estudo foi aprovado pelo Comitê de Ética e Pesquisa do Hospital de Clínicas de Porto Alegre e também foi avaliado e autorizado pela Western Psychological Services (WPS), que detém os direitos autorais da CARS.

\section{Medidas}

Os instrumentos utilizados no estudo são descritos a seguir.

\section{CARS}

É uma escala de 15 itens que auxilia na identificação de crianças com autismo e as distingüe de crianças com prejuízos do desenvolvimento sem autismo. Sua importância consiste na diferenciação do autismo leve-moderado do grave ${ }^{10-}$ 13. É breve e apropriada para uso em qualquer criança acima de 2 anos de idade. Sua construção foi realizada durante 15 anos e incluiu 1.500 crianças autistas. Para tal, levaram-se em conta os critérios diagnósticos de Kanner (1943), Creak (1961), Rutter (1978), Ritvo \& Freeman (1978) e do Diagnostic and Statistical Manual of Mental Disorders (DSM-III) $(1980)^{10}$. A escala avalia o comportamento em 14 domínios geralmente afetados no autismo, mais uma categoria geral de impressão de autismo ${ }^{9-12}$. Estes 15 itens incluem: relações pessoais, imitação, resposta emocional, uso corporal, uso de objetos, resposta a mudanças, resposta visual, resposta auditiva, resposta e uso do paladar, olfato e tato, medo ou nervosismo, comunicação verbal, comunicação não verbal, nível de atividade, nível e consistência da resposta intelectual e impressões gerais. Os escores de cada domínio variam de 1 (dentro dos limites da normalidade) a 4 (sintomas autistas graves). A pontuação varia de 15 a 60 , e o ponto de corte para autismo é $30^{10}$.

O processo de tradução descrito por Sperber ${ }^{15}$ foi utilizado neste estudo, pois se trata de um modelo útil e prático, de escolha para a maioria dos estudos de tradução e adaptação transcultural ${ }^{16,17}$. A CARS foi traduzida do original em inglês para o português, falado no Brasil, por dois tradutores independentes, e as duas versões foram comparadas pelos pesquisadores até a obtenção da versão final, que sofreu retrotradução para o inglês por psiquiatra bilingüe, não participante das etapas anteriores e que não esteve em contato com o texto original. A versão final, chamada CARS-BR, foi aplicada em 60 pacientes para cálculo das propriedades psicométricas, e o teste-reteste foi realizado após um período mínimo de 4 semanas da primeira aplicação, em 50 pacientes. 
Tabela 1 - Características sociais, demográficas e clínicas dos 60 pacientes autistas

\begin{tabular}{lc}
\hline Características & $\mathbf{n}(\mathbf{\%})$ \\
\hline Sexo masculino & $44(73,3)$ \\
Idade (meses) & $111,8 \pm 43,1($ mínimo 36, máximo 204) \\
Procedência & $33(55,0)$ \\
$\quad$ Porto Alegre & $16(26,7)$ \\
Grande Porto Alegre & $11(18,3)$ \\
Interior & \\
Escolaridade & $35(58,3)$ \\
Escola especial & $8(13,4)$ \\
Escola regular & $17(28,3)$ \\
Não freqüenta & $17(28,3)$ \\
Epilepsia associada & \\
\hline
\end{tabular}

\section{ATA}

Desenvolvida por Ballabriga et al., é composta de 23 subescalas, de fácil aplicação, que avaliam o perfil condutual da criança, embasada nos diferentes aspectos diagnósti$\cos ^{18}$. A escala permite seguimentos longitudinais de evolução e suas características psicométricas, em português, foram satisfatórias ${ }^{19}$. Além disso, a ATA é um questionário de screening para tentar diferenciar autistas de deficientes mentais sem autismo. Seu ponto de corte é 15 . Foi utilizada neste estudo com objetivo de obter a validade convergente da CARS-BR.

\section{GAF}

Trata-se de uma escala de 100 pontos cujo principal objetivo é fornecer um escore capaz de refletir o nível global de funcionamento do paciente. Esta escala pode ser utilizada para planejar e medir o impacto do tratamento, seguir as mudanças do paciente ao longo do tempo, avaliar qualidade de vida e estimar o prognóstico. Pode ser utilizada em qualquer situação em que uma avaliação de gravidade é necessária $^{20}$. Neste estudo, foi utilizada a fim de obter a validade discriminante da CARS-BR.

\section{Análise estatística}

Primeiramente, foi utilizado o alfa de Cronbach para avaliação da consistência interna. O coeficiente de correlação de Pearson foi utilizado para avaliação da validade convergente e validade discriminante. A confiabilidade teste-reteste foi obtida através do cálculo do nível de significância de $5 \%$. Todos os dados foram analisados através do programa estatístico SPSS 12.0.

\section{Resultados}

Na amostra estudada, houve predomínio do sexo masculino, e a média de idade foi de 111,8 meses ( 9,3 anos). Aproximadamente $55 \%$ dos pacientes eram procedentes de Porto
Alegre, e 58,3\% tinham acesso à escola especial para atendimento de crianças com transtorno invasivo do desenvolvimento.

As características sociais e demográficas do grupo estudado são apresentadas na Tabela 1.

A versão da CARS em português encontra-se na Tabela 2.

Aproximadamente $65 \%$ (39) dos pacientes avaliados encontravam-se na categoria de autismo grave, e 32\% na categoria de leve a moderado. O restante $(3 \%)$, segundo a CARS-BR, não apresentava autismo.

A associação entre autismo infantil e epilepsia é bem conhecida e, neste estudo, esteve presente em $28,3 \%$ dos pacientes avaliados.

\section{Propriedades psicométricas}

\section{Consistência interna}

A média ( \pm SD) do total de pontos obtidos foi 39,4 ( \pm $5,07)$. Na análise da consistência interna da escala utilizando o coeficiente alfa de Cronbach, obteve-se o valor 0,82 (IC95\% $0,71-0,88)$, indicando um elevado grau de consistência interna.

\section{Validade convergente}

A associação observada entre a CARS e a ATA foi expressa por um coeficiente de Pearson $r=0,89$ (IC95\% 0,74-0,90); $\mathrm{p}<0,001$ (Figura 1).

\section{Validade discriminante}

Conforme esperado, houve uma correlação inversa significativa entre a CARS e GAF: $r=-0,75$ (IC95\% -0,84-0,61); $\mathrm{p}<0,001$ (Figura 2).

\section{Confiabilidade teste-reteste}

Após um período mínimo de 4 semanas, 50 pacientes foram novamente avaliados. A análise, através do coeficiente kappa de Cohen, mostrou concordância de 0,90. 0 
Tabela 2 - Versão em português da CARS

I. Relações pessoais: 1 Nenhuma evidência de dificuldade ou anormalidade nas relações pessoais: O comportamento da criança é adequado à sua idade. Alguma timidez, nervosismo ou aborrecimento podem ser observados quando é dito à criança o que fazer, mas não em grau atípico; 2 Relações levemente anormais: A criança pode evitar olhar o adulto nos olhos, evitar o adulto ou ter uma reação exagerada se a interação é forçada, ser excessivamente tímida, não responder ao adulto como esperado ou agarrar-se ao pais um pouco mais que a maioria das crianças da mesma idade; 3 Relações moderadamente anormais: Às vezes, a criança demonstra indiferença (parece ignorar o adulto). Outras vezes, tentativas persistentes e vigorosas são necessárias para se conseguir a atenção da criança. O contato iniciado pela criança é mínimo; 4 Relações gravemente anormais: A criança está constantemente indiferente ou inconsciente ao que o adulto está fazendo. Ela quase nunca responde ou inicia contato com o adulto. Somente a tentativa mais persistente para atrair a atenção tem algum efeito.

II. Imitação: 1 Imitação adequada: A criança pode imitar sons, palavras e movimentos, os quais são adequados para o seu nível de habilidade; 2 Imitação levemente anormal: Na maior parte do tempo, a criança imita comportamentos simples como bater palmas ou sons verbais isolados; ocasionalmente imita somente após estimulação ou com atraso; 3 Imitação moderadamente anormal: A criança imita apenas parte do tempo e requer uma grande dose de persistência ou ajuda do adulto; freqüentemente imita apenas após um tempo (com atraso); 4 Imitação gravemente anormal: A criança raramente ou nunca imita sons, palavras ou movimentos mesmo com estímulo e assistência.

III. Resposta emocional: 1 Resposta emocional adequada à situação e à idade: A criança demonstra tipo e grau adequados de resposta emocional, indicada por uma mudança na expressão facial, postura e conduta; 2 Resposta emocional levemente anormal: A criança ocasionalmente apresenta um tipo ou grau inadequados de resposta emocional. Às vezes, suas reações não estão relacionadas a objetos ou a eventos ao seu redor; 3 Resposta emocional moderadamente anormal: A criança demonstra sinais claros de resposta emocional inadequada (tipo ou grau). As reações podem ser bastante inibidas ou excessivas e sem relação com a situação; pode fazer caretas, rir ou tornar-se rígida até mesmo quando não estejam presentes objetos ou eventos produtores de emoção; 4 Resposta emocional gravemente anormal: As respostas são raramente adequadas à situação. Uma vez que a criança atinja um determinado humor, é muito difícil alterá-lo. Por outro lado, a criança pode demonstrar emoções diferentes quando nada mudou.

IV. Uso corporal: 1 Uso corporal adequado à idade: A criança move-se com a mesma facilidade, agilidade e coordenação de uma criança normal da mesma idade; 2 Uso corporal levemente anormal: Algumas peculiaridades podem estar presentes, tais como falta de jeito, movimentos repetitivos, pouca coordenação ou a presença rara de movimentos incomuns; 3 Uso corporal moderadamente anormal:

Comportamentos que são claramente estranhos ou incomuns para uma criança desta idade podem incluir movimentos estranhos com os dedos, postura peculiar dos dedos ou corpo, olhar fixo, beliscar o corpo, auto-agressão, balanceio, girar ou caminhar nas pontas dos pés; 4 Uso corporal gravemente anormal: Movimentos intensos ou freqüentes do tipo listado acima são sinais de uso corporal gravemente anormal. Estes comportamentos podem persistir apesar das tentativas de desencorajar as crianças a fazê-los ou de envolver a criança em outras atividades.

V. Uso de objetos: 1 Uso e interesse adequados por brinquedos e outros objetos: A criança demonstra interesse normal por brinquedos e outros objetos adequados para o seu nível de habilidade e os utiliza de maneira adequada; 2 Uso e interesse levemente inadequados por brinquedos e outros objetos: A criança pode demonstrar um interesse atípico por um brinquedo ou brincar com ele de forma inadequada, de um modo pueril (exemplo: batendo ou sugando o brinquedo); 3 Uso e interesse moderadamente inadequados por brinquedos e outros objetos: A criança pode demonstrar pouco interesse por brinquedos ou outros objetos, ou pode estar preocupada em usá-los de maneira estranha. Ela pode concentrar-se em alguma parte insignificante do brinquedo, tornar-se fascinada com a luz que reflete do mesmo, repetitivamente mover alguma parte do objeto ou exclusivamente brincar com ele; 4 Uso e interesse gravemente inadequados por brinquedos e outros objetos: A criança pode engajar-se nos mesmos comportamentos citados acima, porém com maior freqüência e intensidade. É difícil distrair a criança quando ela está engajada nestas atividades inadequadas.

VI. Resposta a mudanças: 1 Respostas à mudança adequadas à idade: Embora a criança possa perceber ou comentar as mudanças na rotina, ela é capaz de aceitar estas mudanças sem angústia excessiva; 2 Respostas à mudança adequadas à idade levemente anormal: Quando um adulto tenta mudar tarefas, a criança pode continuar na mesma atividade ou usar os mesmos materiais; 3 Respostas à mudança adequadas à idade moderadamente anormal: A criança resiste ativamente a mudanças na rotina, tenta continuar sua antiga atividade e é difícil de distraí-la. Ela pode tornar-se infeliz e zangada quando uma rotina estabelecida é alterada; 4 Respostas à mudança adequadas à idade gravemente anormal: A criança demonstra reações graves às mudanças. Se uma mudança é forçada, ela pode tornar-se extremamente zangada ou não disposta a ajudar e responder com acessos de raiva

VII. Resposta visual: 1 Resposta visual adequada: O comportamento visual da criança é normal e adequado para sua idade. A visão é utilizada em conjunto com outros sentidos como forma de explorar um objeto novo; 2 Resposta visual levemente anormal: A criança precisa, ocasionalmente, ser lembrada de olhar para os objetos. A criança pode estar mais interessada em olhar espelhos ou luzes do que o fazem seus pares, pode ocasionalmente olhar fixamente para o espaço, ou pode evitar olhar as pessoas nos olhos; 3 Resposta visual moderadamente anormal: A criança deve ser lembrada freqüentemente de olhar para o que está fazendo, ela pode olhar fixamente para o espaço, evitar olhar as pessoas nos olhos, olhar objetos de um ângulo incomum ou segurar os objetos muito próximos aos olhos; 4 Resposta visual gravemente anormal: A criança evita constantemente olhar para as pessoas ou para certos objetos e pode demonstrar formas extremas de outras peculiaridades visuais descritas acima. 
Tabela 2 - Versão em português da CARS (cont.)

VIII. Resposta auditiva : 1 Respostas auditivas adequadas para a idade: O comportamento auditivo da criança é normal e adequado para idade. A audição é utilizada junto com outros sentidos; 2 Respostas auditivas levemente anormais: Pode haver ausência de resposta ou uma resposta levemente exagerada a certos sons. Respostas a sons podem ser atrasadas e os sons podem necessitar de repetição para prender a atenção da criança. A criança pode ser distraída por sons externos; 3 Respostas auditivas moderadamente anormais: As respostas da criança aos sons variam. Freqüentemente ignora o som nas primeiras vezes em que é feito. Pode assustar-se ou cobrir as orelhas ao ouvir alguns sons do cotidiano; 4 Respostas auditivas gravemente anormais: A criança reage exageradamente e/ou despreza sons num grau extremamente significativo, independente do tipo de som

IX. Resposta e uso do paladar, olfato e tato: 1 Uso e resposta normais do paladar, olfato e tato: A criança explora novos objetos de um modo adequado a sua idade, geralmente sentindo ou olhando. Paladar ou olfato podem ser usados quando adequados. Ao reagir a pequenas dores do dia a dia, a criança expressa desconforto, mas não reage exageradamente; 2 Uso e resposta levemente anormais do paladar, olfato e tato: A criança pode persistir em colocar objetos na boca; pode cheirar ou provar/experimentar objetos não comestíveis. Pode ignorar ou ter reação levemente exagerada à uma dor mínima, para a qual uma criança normal expressaria somente desconforto; 3 Uso e resposta moderadamente anormais do paladar, olfato e tato: A criança pode estar moderadamente preocupada em tocar, cheirar ou provar objetos ou pessoas. A criança pode reagir demais ou muito pouco; 4 Uso e resposta gravemente anormais do paladar, olfato e tato: $\mathrm{A}$ criança está preocupada em cheirar, provar e sentir objetos, mais pela sensação do que pela exploração ou uso normal dos objetos. A criança pode ignorar completamente a dor ou reagir muito fortemente a desconfortos leves.

X. Medo ou nervosismo: 1 Medo ou nervosismo normais: O comportamento da criança é adequado tanto à situação quanto à idade; 2 Medo ou nervosismo levemente anormais: A criança ocasionalmente demonstra muito ou pouco medo ou nervosismo quando comparada às reações de uma criança normal da mesma idade e em situação semelhante; 3 Medo ou nervosismo moderadamente anormais: A criança demonstra bastante mais ou bastante menos medo do que seria típico para uma criança mais nova ou mais velha em uma situação similar; 4 Medo ou nervosismo gravemente anormais: Medos persistem mesmo após experiências repetidas com eventos ou objetos inofensivos. É extremamente difícil acalmar ou confortar a criança. A criança pode, por outro lado, falhar em demonstrar consideração adequada aos riscos que outras crianças da mesma idade evitam.

XI. Comunicação verbal: 1 Comunicação verbal normal, adequada à idade e à situação; 2 Comunicação verbal levemente anormal: A fala demonstra um atraso global. A maior parte do discurso tem significado; porém, alguma ecolalia ou inversão pronominal podem ocorrer. Algumas palavras peculiares ou jargões podem ser usados ocasionalmente; 3 Comunicação verbal moderadamente anormal: A fala pode estar ausente. Quando presente, a comunicação verbal pode ser uma mistura de alguma fala significativa e alguma linguagem peculiar, tais como jargão, ecolalia ou inversão pronominal. As peculiaridades na fala significativa podem incluir questionamentos excessivos ou preocupação com algum tópico em particular; 4 Comunicação verbal gravemente anormal: Fala significativa não é utilizada. A criança pode emitir gritos estridentes e infantis, sons animais ou bizarros, barulhos complexos semelhantes à fala, ou pode apresentar o uso bizarro e persistente de algumas palavras reconhecíveis ou frases

XII. Comunicação não-verbal: 1 Uso normal da comunicação não-verbal adequado à idade e situação; 2 Uso da comunicação não-verbal levemente anormal: Uso imaturo da comunicação não-verbal; a criança pode somente apontar vagamente ou esticar-se para alcançar o que quer, nas mesmas situações nas quais uma criança da mesma idade pode apontar ou gesticular mais especificamente para indicar o que deseja; 3 Uso da comunicação não-verbal moderadamente anormal: A criança geralmente é incapaz de expressar suas necessidades ou desejos de forma não verbal, e não consegue compreender a comunicação não-verbal dos outros; 4 Uso da comunicação não-verbal gravemente anormal: A criança utiliza somente gestos bizarros ou peculiares, sem significado aparente, e não demonstra nenhum conhecimento do significados associados aos gestos ou expressões faciais dos outros.

XIII. Nível de atividade: 1 Nível de atividade normal para idade e circunstâncias: A criança não é nem mais nem menos ativa que uma criança normal da mesma idade em uma situação semelhante; 2 Nível de atividade levemente anormal: A criança pode tanto ser um pouco irrequieta quanto um pouco "preguiçosa", apresentando, algumas vezes, movimentos lentos. O nível de atividade da criança interfere apenas levemente no seu desempenho; 3 Nível de atividade moderadamente anormal: A criança pode ser bastante ativa e difícil de conter. Ela pode ter uma energia ilimitada ou pode não ir prontamente para a cama à noite. Por outro lado, a criança pode ser bastante letárgica e necessitar de um grande estímulo para mover-se; 4 Nível de atividade gravemente anormal: A criança exibe extremos de atividade ou inatividade e pode até mesmo mudar de um extremo ao outro.

XIV. Nível e consistência da resposta intelectual: $1 \mathrm{~A}$ inteligência é normal e razoavelmente consistente em várias áreas: A criança é tão inteligente quanto crianças típicas da mesma idade e não tem qualquer habilidade intelectual ou problemas incomuns; 2 Funcionamento intelectual levemente anormal: A criança não é tão inteligente quanto crianças típicas da mesma idade; as habilidades apresentam-se razoavelmente regulares através de todas as áreas; 3 Funcionamento intelectual moderadamente anormal: Em geral, a criança não é tão inteligente quanto uma típica criança da mesma idade, porém a criança pode funcionar próximo do normal em uma ou mais áreas intelectuais; 4 Funcionamento intelectual gravemente anormal: Embora a criança geralmente não seja tão inteligente quanto uma criança típica da mesma idade, ela pode funcionar até mesmo melhor que uma criança normal da mesma idade em uma ou mais áreas.

XV. Impressões gerais: 1 Sem autismo: a criança não apresenta nenhum dos sintomas característicos do autismo; 2 Autismo leve: A criança apresenta somente um pequeno número de sintomas ou somente um grau leve de autismo; 3 Autismo moderado: A criança apresenta muitos sintomas ou um grau moderado de autismo; 4 Autismo grave: a criança apresenta inúmeros sintomas ou um grau extremo de autismo.

Pode ser pontuada utilizando valores intermediários $=1,5 ; 2,5 ;$ e 3,5 .

15-30 = sem autismo 30-36 = autismo leve-moderado 36-60 = autismo grave 


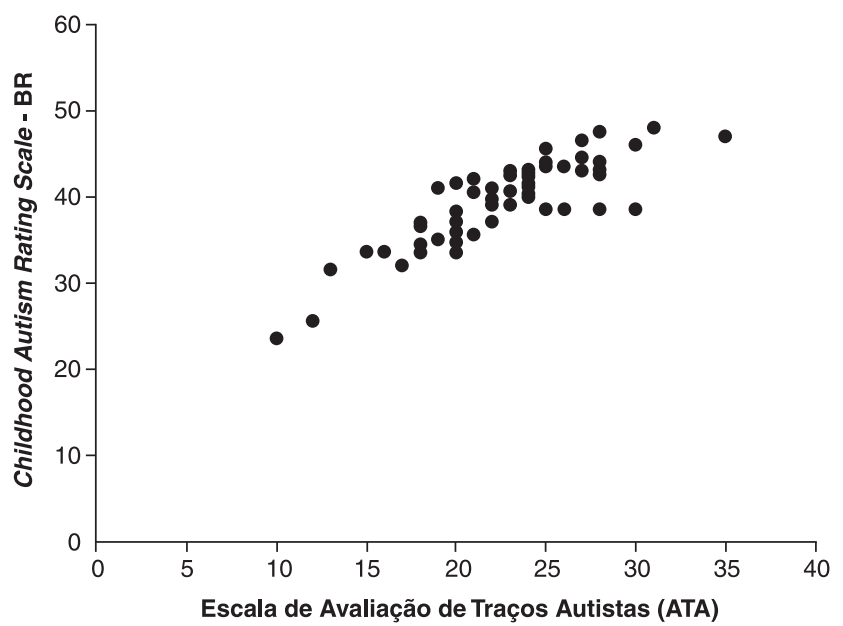

Figura 1 - Correlação entre Childhood Autism Rating Scale (CARSBR) e Avaliação de Traços Autistícos (ATA) $(n=60)$

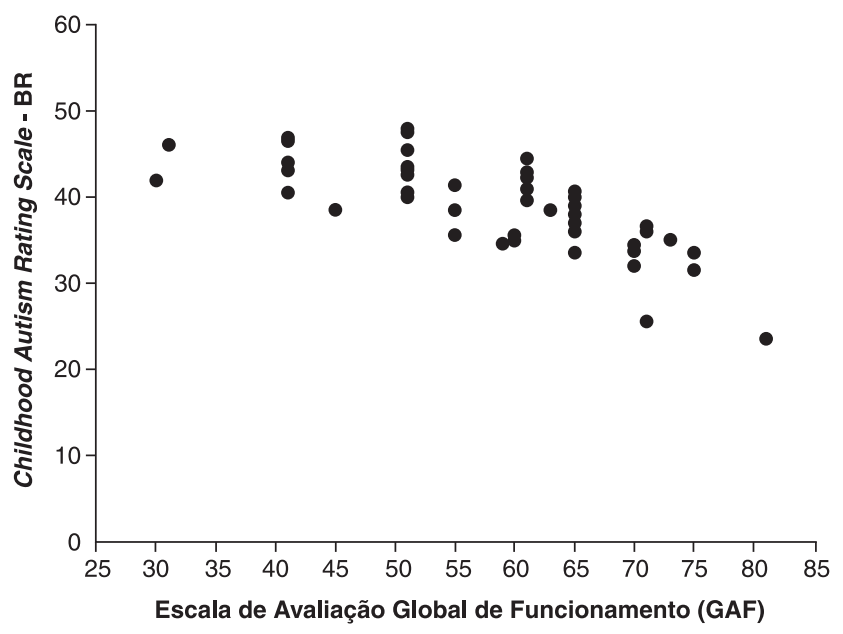

Figura 2 - Correlação entre Childhood Autism Rating Scale (CARSBR) e Escala de Avaliação Global do Funcionamento ( $n=$ 60)

resultado é uma indicação da estabilidade da escala ao longo do tempo, não sendo necessário reaplicar a escala nos 60 pacientes iniciais ${ }^{1}$.

\section{Discussão}

Desde sua descrição, há mais de 60 anos, o autismo representa um desafio fascinante e enigmático para neurologistas e psiquiatras ${ }^{5}$. Sabe-se hoje que o autismo não é uma doença única, e sim um distúrbio de desenvolvimento complexo associado a múltiplas etiologias e a graus variados de severidade, sendo caracterizado por alterações comportamentais, de linguagem e de cognição, com retardo mental em $70 \%$ dos casos e crises epilépticas em $30 \%{ }^{6,7}$. Não há duvida da importância dos fatores biológicos na gênese do autismo, porém, não havendo um marcador, seu diagnóstico e o conhecimento de seus limites permanecem uma decisão clínica ${ }^{3,7}$ e, portanto, o uso de testes padronizados para avaliação do transtorno é de considerável interesse na comunidade científica ${ }^{14}$.

Nosso estudo traduziu e validou para a língua portuguesa, do Brasil, a CARS, uma escala mundialmente utilizada para diagnóstico e classificação do autismo e que, pela sua importância, já está traduzida para o japonês, sueco, francês, entre outros idiomas ${ }^{21-23}$. A CARS-BR apresentou boa consistência interna, validade discriminante, validade convergente e confiabilidade teste-reteste, utilizando uma amostra de crianças e adolescentes com autismo tratados em regime ambulatorial em um hospital terciário. Estes resultados são comparáveis ao da escala original e às outras versões. A confiabilidade é a reproducibilidade de uma medida e pode ser avaliada de várias formas: confiabilidade teste-reteste, que é o grau de concordância entre as avaliações em momentos diferentes e pode ser estimada pelo coeficiente kappa, confiabilidade entre diferentes observadores e consistência interna ${ }^{14,24}$. A confiabilidade entre observadores mereceu atenção em diversos estudos envolvendo a CARS, mas os resultados são difíceis de avaliar e comparar ${ }^{22}$ e, portanto, não foi utilizada neste estudo. A consistência interna representa o grau no qual a escala, vista como um todo, mede um fenômeno isolado e é medida através do coeficiente alfa de Cronba$\mathrm{ch}^{22,24}$. As medidas de validade estão relacionadas aos achados de confiabilidade e são o aspecto mais importante da avaliação psicométrica ${ }^{14,22}$. Podem ser definidas como a capacidade em realmente medir aquilo a que o instrumento se propõe e inclui a validade de critério, de conteúdo e de construção (convergente, divergente e discriminante) ${ }^{24}$.

As características psicométricas da versão em português da CARS são semelhantes às da amostra que deu origem ao instrumento ${ }^{10}$. A literatura, de um modo geral, sustenta a confiabilidade da CARS com vários estudos demonstrando uma consistência interna com valores aceitáveis $\geq 0,90^{13,22,25}$. A consistência interna da CARS-BR, medida pelo coeficiente a de Cronbach, é considerada boa $(0,83)$, assim como na escala original $(0,94)$, e justifica a combinação de 15 itens individuais em um único escore ${ }^{10,24}$. O valor do alfa de Cronbach na versão sueca foi $0,91^{22}$ e, na versão japonesa, de 0,8721,25. Após o paciente ter sido avaliado para cada um dos 15 itens, foi calculado um escore total. Aproximadamente $32 \%$ (19) dos pacientes deste estudo foram incluídos na categoria de autismo leve a moderado, e 65\% (39) dos pacientes apresentavam critérios de autismo grave. Estes resultados podem ser explicados pelo fato de a amostra ser proveniente de um hospital universitário com atendimento especializado na área. $\mathrm{O}$ DSM-IV representa um sistema de classificação desenvolvido pela Associação Americana de Psiquiatria ${ }^{4}$ e utiliza as três características básicas do autismo, enquanto os 15 itens da CARS permitem um diagnóstico mais objetivo ao incluirem as características de autismo primário descritas por Kanner, as observadas por Creak \& Rutter e escalas adicionais (CID-10 e DSM-IV $)^{10}$. A correspondência entre os dois métodos pode chegar a $98 \%{ }^{9,11}$, sendo, portanto, complementares no diagnóstico. 
A maioria dos instrumentos de avaliação elaborada para pesquisa médica teve sua origem em países ocidentais desenvolvidos e foi baseada em conceitos, formatos, normas e expectativas prevalentes nesses países ${ }^{15,26}$. Na maioria dos casos, tem-se optado pela tradução e adaptação transcultural de escalas já existentes, pois se trata de um procedimento mais prático que o desenvolvimento de uma escala original, além de permitir a comparação de resultados entre diferentes países ${ }^{15-17,27}$.

O método utilizado nas traduções de instrumentos para línguas e culturas diferentes tem sido amplamente discutido, pois como muitos dos instrumentos vêm sendo utilizados em realidades socioculturais distintas daquelas de onde se originaram, a questão fundamental é se podemos inferir que os escores resultantes dessas avaliações têm o mesmo significado para populações etnoculturais diferentes ${ }^{26,27}$. Perneger et al. ${ }^{28}$ investigaram as características de duas versões de instrumentos de qualidade de vida traduzidas por métodos diferentes e concluíram que a versão obtida através de processo exaustivo apresentou as mesmas características psicométricas de um método mais simples, sugerindo que um processo menos rebuscado não compromete a qualidade do instrumento final. A tradução e adaptação transcultural de qualquer escala na área da saúde requerem cuidados lingüísticos, e a importância em se buscar equivalência entre a versão original e a versão em português tem sido cada vez mais reconhecida, principalmente em um país com as dimensões do Brasil, em que predominam as diferenças regionais e de escolaridade $^{27}$. Mattos, ao apresentar uma versão em português do instrumento MTA-SNAP-IV de avaliação de sintomas de transtorno do déficit de atenção/hiperatividade e sintomas de transtorno desafiador e de oposição, ressalta a complexidade em se aplicar instrumentos em culturas distintas daquelas para as quais foram criados. O autor também aponta para a utilização de uma metodologia que inclua tradução, retrotradução, análise das versões e aplicação em população-alvo ${ }^{27}$.

O principal objetivo deste trabalho foi determinar as características psicométricas iniciais da versão em português da CARS, mas, além destes resultados, nossos dados também demonstraram uma elevada associação entre autismo e epilepsia (28,3\%), consistente com estudos específicos prévios que relatam uma taxa de epilepsia em crianças com autismo de 5 a $39 \%{ }^{29}$. Embora todos estes resultados sejam positivos, estudos adicionais são necessários para complementar os dados obtidos. Nossos achados, apesar da concordância com os dados da literatura, devem ser interpretados com cautela, principalmente em função do número de pacientes da amostra. Trata-se de uma aplicação inicial do instrumento e variáveis culturais regionais, assim como diferenças socioculturais devem ser estudadas de modo mais amplo. A metodologia utilizada, os cuidados no processo de tradução e a avaliação psicométrica da versão em português permitem concluir que este instrumento é válido e confiável para avaliação da gravidade do autismo em crianças brasileiras. O presente estudo representa o primeiro passo visando um melhor diagnóstico do autismo em nosso meio e possibilitará, no futuro, a aplicação da CARS-BR nas cinco regiões do Brasil com posterior comparação dos resultados.

\section{Agradecimentos}

Os autores agradecem ao Dr. Andre Palmini pela leitura crítica do artigo.

\section{Referências}

1. Ajuriahuerra J. Manual de psiquiatria infantil. $4^{a}$ ed. Barcelona: Toray-Masson; 1977.

2. Kanner L. Autistic disturbances of affective contact. Nerv Child.1943;2:217-50.

3. Gadia C, Tuchman R, Rotta NT. Autismo e doenças invasivas de desenvolvimento. J Pediatr (Rio J). 2004;80:S83-94.

4. American Psychiatric Association, editor. Diagnostic and statistical manual of mental disorders - DSM-IV. 4th ed. Washington, DC: American Psychiatric Association; 1994. p. 65-78.

5. Ozand PT, Al Odaib A, Merza H, Al Harbi S. Autism: a review. J Pediatr Neurol. 2003;1:55-67.

6. Dawson G, Webb S, Schellenberg GB, et al. Defining the broader phenotype of autism: genetic, brain, and behavioral perspectives. Dev Psychopathol. 2002; 14:581-611.

7. Minshew NJ, Williams DL. The new neurobiology of autism: cortex, connectivity, and neuronal organization. Arch Neurol. 2007;64:945-50.

8. Rutter M. Incidence of autism spectrum disorders: changes over time and their meaning. Acta Paediatr. 2005;94:2-15.

9. Rellini E, Tortolani D, Trillo S, Carbone S, Montecchi F. Childhood Autism Rating Scale (CARS) and Autism Behavior Checklist (ABC) correspondence and conflicts with DSM-IV criteria in diagnosis of autism. J Autism Dev Disord. 2004;34:703-8.

10. Schopler E, Reichler R, Renner BR. The Childhood Autism Rating Scale (CARS). 10th ed. Los Angeles, CA: Western Psychological Services; 1988.

11. Eaves RC, Milner B. The criterion-related validity of the Childhood Autism Rating Scale and the Autism Behavior Checklist. J Abnorm Child Psychol. 1993;21:481-91.

12. Stella J, Mundy P, Tuchman R. Social and nonsocial factors in the Childhood Autism Rating Scale. J Autism Dev Disord. 1999; 29:307-17.

13. Magyar CI, Pandolfi V. Factor structure evaluation of the childhood autism rating scale. J Autism Dev Disord. 2007; 37:1787-94.

14. Matson JL, Nebel-Schwalm M, Matson ML. A review of methodological issues in the differential diagnosis of autism spectrum disorders in children. Res Autism Spectr Discord. 2006; $1: 38-54$

15. Sperber AD. Translation and validation of study instruments for cross-cultural research. Gastroenterology. 2004;126:S124-8.

16. Alievi PT, Carvalho PR, Trotta EA, Mombelli Filho R. The impact of admission to a pediatric intensive care unit assessed by means of global and cognitive performance scales. J Pediatr (Rio J). 2007;83:505-11. 
17. Guillemin F, Bombardier C, Beaton D. Cross-cultural adaptation of health-related quality of life measures: literature review and proposed guidelines. J Clin Epidemiol.1993;46:1417-32.

18. Ballabriga MC, Escudé RM, Llaberia ED. Escala d'avaluació dels trests autistes (ATA): validez y fiabilidad de una escala para el examen de las conductas autistas. Rev Psiquiatr Infanto-Juvenil. 1994;4:254-63.

19. Assumpção Jr FB, Kuczynski E, Gabriel MR, Rocca CC. Escala de avaliação de traços autísticos (ATA): validade e confiabilidade de uma escala para a deteç̧ão de condutas autísticas. Arq Neuro-psiquiatr.1999;57:23-9.

20. Spitzer RL, Gibbon M, Endicott J. Global Assessment Scale (GAS), Global Assessment of Functioning (GAF) Scale, Social and Occupational Functioning Assessment Scale (SOFAS). In: Rush $\mathrm{AJ}$, editor. Handbook of psychiatric measures. Washington: American Psychiatric Association; 2000. p. 96-100.

21. Kurita $H$, Miyake $Y$, Katsuno $K$. Reliability and validity of the Childhood Autism Rating Scale-Tokyo Version (CARS-TV). J Autism Dev Disord.1989;19:389-96.

22. Nordin V, Gillberg C, Nyden A. The Swedish version of the Childhood Autism Rating Scale in a clinical setting. J Autism Dev Disord. 1998;28:69-75.

23. Pry R, Aussilloux C. Le Childhood Autism Rating Scale (CARS) chez I' enfant autiste jeune: analyse des items, étude des traits latents, validité concourante et généralisabilité. Psychologie et psychometrie. 2000;21:33-47.
24. Blacker D, Endicott J. Psychometric properties: concepts of reliability and validity. In: Rush AJ, editor. Handbook of psychiatric measures. Washington, DC: American Psychiatric Association; 2000. p. 7-14.

25. Tachimori $\mathrm{H}$, Osada $\mathrm{H}$, Kurita $\mathrm{H}$. Childhood autism rating scale-Tokyo version for screening pervasive developmental disorders. Psychiatry Clin Neurosci. 2003;57:113-29.

26. Jorge MR. Adaptação transcultural de instrumentos de pesquisa em saúde mental. Rev Psiq Clin.1998;25:233-9.

27. Mattos $P$, Serra-Pinheiro MA, Rohde LA, Pinto D. A Brazilian version of the MTA-SNAP-IV for evaluation of symptoms of attention deficit/ hyperactivity disorder and oppositional-defiant disorder. Rev Psiquiatr Rio Gd Sul. 2006;28:290-7.

28. Perneger TV, Leplege A, Etter JF. Cross-cultural adaptation of a psychometric instrument: two methods compared. J Clin Epidemiol. 1999;52:1037-46.

29. Tuchman R, Rapin I. Epilepsy in autism. Lancet Neurol. 2002; $1: 352-8$.

Correspondência:

Alessandra M. Pereira

Alameda Eduardo Guimarães, 73/902

CEP 91340-350 - Porto Alegre, RS

E-mail: ampereirabr@yahoo.com.br 